\title{
Representações sociais dos conselheiros municipais acerca do controle social em saúde no SUS
}

\author{
Social representations of municipal counselors regarding social \\ control in health in the Brazilian Unified Health System (SUS)
}

Helena Eri Shimizu ${ }^{1}$

Marcio Florentino Pereira ${ }^{1}$

Antonio José Costa Cardoso ${ }^{1}$

Ximena Pamela Claudia Dias Bermudez ${ }^{1}$

${ }^{1}$ Departamento de Saúde Coletiva, Universidade de Brasília. SQN 205 Bloco G/ 605, Asa Norte. 70.843-070 Brasília DF.

shimizu@unb.br

\begin{abstract}
This study seeks to analyze the social representations of municipal health counselors regarding social control in health. Sixty interviews were conducted with counselors of nineteen counties within the Integrated Federal District Region and Surrounding Areas (RIDE-DF). Data analysis was conducted with the use of French Alceste software, which included two thematic groups. The first dealt with the weaknesses of health councils, consisting of four categories: limitations in decision-making powers, bureaucratization of everyday practices, weaknesses in social participation and limitations in the practice of representation. The second dealt with the health system in RIDE-DF, consisting of only one category that expresses the precarious organization of health care in RIDE-DF. Social representation of social control, with a focus on social participation appears to be constrained by a ritual, namely the bureaucratization of policies and practices in the councils. This form of hierarchical and bureaucratic organization and operation of the council, based on a centralizing management model, has distanced the counselors from discussing health needs geared to the construction of public Health Projects.
\end{abstract}

Key words Health counseling, Social control, Participative management, Democracy
Resumo Este estudo tem como objetivo analisar as representações sociais dos conselheiros municipais de saúde acerca do controle social em saúde. Foram realizadas 60 entrevistas com os conselheiros de dezenove municípios da Região Integrada do Distrito Federal e Entorno (RIDE-DF). A análise foi feita com o software Analyse Lexicale par Context d' um Essemble de Segments de Texte (ALCESTE) que apreendeu dois eixos temáticos: o primeiro, que trata das fragilidades dos conselhos de saúde, constituidas por quatro classes (as limitações no poder de decisão, a burocratização das práticas cotidianas, as fragilidades da participação social; as limitações na prática da representação); e o segundo, que trata do sistema de saúde na RIDE-DF, constituído por apenas uma classe que expressa à precária organização da atenção à saúde na RIDE-DF. A representação social do controle com foco na participação social parece ser constrangida por um ritual - o da burocratização das políticas e práticas nos conselhos. Essa forma de organização e funcionamento dos conselhos, hierarquizada e burocratizada, ancoradas em modelos de gestão centralizadora, têm distanciado os conselheiros das discussões sobre as necessidades de saúde, voltados para a construção de Projeto de Saúde coletivos.

Palavras-chave Conselhos de saúde, Controle social, Gestão participativa, Democracia 


\section{Introdução}

A criação dos conselhos de saúde (CS) como instância institucionalizada de participação social, sem dúvida, significa uma conquista da sociedade civil em busca da construção de uma perspectiva mais democrática em saúde ${ }^{1-3}$. Em realidade, espera-se que os CS possibilitem novas formas de interação entre governo e sociedade, que resultem em novos padrões de governo baseados na gestão democrática, com centro em três pilares fundamentais: maior responsabilidade dos governos em formular políticas públicas consentâneas com as necessidades da população; reconhecimento dos direitos sociais; e abertura de canais para ampla participação da sociedade ${ }^{3-5}$.

Os CS podem alcançar uma democracia de alta intensidade se contemplarem satisfatoriamente as seguintes dimensões: a partilha de poder, a reciprocidade e o reconhecimento ${ }^{5}$. Ressalte-se, a democracia representativa tende a ser uma democracia de baixa intensidade pois, ao definir de modo restritivo o espaço público, deixa intactas muitas relações de poder que não transforma em autoridade partilhada; ao assentar em ideais de igualdade formal e não real, não garante a realização das condições que a tornam possível ${ }^{5}$.

Há que se considerar, também, que a concretização de suas proposições depende, sobretudo, da forma como funcionam os núcleos de poder e da maneira que se realizam as interações entre os diversos segmentos representantes no conselho, ou seja, do governo, do prestador, do trabalhador e do usuário ${ }^{1}$.

A instituição de práticas de gestão democráticas nos municípios está sujeito à forma como os conselhos de saúde se organizam para bloquear ou minimizar práticas clientelistas e a captura das esferas públicas por interesses corporativos; desenvolver práticas e estruturas horizontais de participação, capazes de gerar sujeitos políticos autônomos; e estimular e reforçar os vínculos associativos dos grupos locais.

Assim sendo, buscou-se analisar, neste estudo, a intensidade democrática dos conselhos de saúde à luz da Teoria das Representações Sociais, por permitir compreender a natureza das relações e interações sociais, com suas dinâmicas e clarificar os processos das práticas sociais ${ }^{6}$.

A representação social, mais do que uma algo compartilhado da realidade, é uma organização significante dos atores diretamente envolvidos, seja individual ou coletivamente, com a práxis da mudança e da transformação. Nessa perspectiva, compreende-se que as representações sociais contribuem também para a formação das identidades nos indivíduos e grupos, tendo por função situá-los dentro do campo social ${ }^{7}$.

Assim, neste estudo pretendeu-se conhecer quais são as representações sociais dos conselheiros acerca do controle social em saúde, bem como identificar as potencialidades e dificuldades desse espaço de cogestão do SUS, que tem como finalidade ampliar a participação democrática.

\section{Metodologia}

O estudo foi realizado em dezenove conselhos municipais da Região Integrada do Distrito Federal e Entorno (RIDE-DF): Águas Lindas de Goiás, Cidade Ocidental, Cristalina, Luziânia, Novo Gama, Santo Antônio do Descoberto, Valparaíso de Goiás (Região do Entorno Sul), Buritis, Cabeceira Grande e Unaí (Região de Unaí), Abadiânia, Colcalzinho, Padre Bernardo e Pirenópolis (Região dos Pirineus), Formosa, Água Fria, Planaltina de Goiás e Vila Boa (Região do Entorno Norte).

Foram feitas 60 entrevistas com conselheiros de saúde, com pelo menos três meses de atuação no CS, representantes dos segmentos: dezoito dos gestores, dezesseis dos trabalhadores, dezessete dos usuários e nove dos prestadores de dezenove municípios da RIDE-DF.

Utilizou-se um roteiro semiestruturado para a entrevista com os seguintes tópicos:

. Fale sobre participação social e controle social;

. Fale sobre organização e funcionamento do Conselho Municipal de Saúde;

. Comente sobre a participação do Conselho na elaboração do plano municipal de Saúde; e

. Fale sobre participação do conselho nas ações de monitoramento e avaliação das ações de saúde.

A coleta de dados ocorreu no período de 30 janeiro de 2010 a março de 2011. O Projeto de pesquisa foi aprovado pelo Comitê de Ética da Faculdade de Ciências da Saúde.

Foi utilizado, para análise dos dados, o software Analyse Lexicale par Context d'un Essemble de Segments de Texte (ALCESTE), que capta trechos do discurso, aparentemente diferentes em seu enunciado, mas próximas em uma relação de significado para um determinado grupo social. Em outras palavras, o software coloca em destaque aglomerados (mundos lexicais) de palavras (palavras-plenas) que têm por referência um mesmo núcleo de sentido ${ }^{8,9}$. 
A associação de palavras portadoras de sentido que aparecem com frequência constituem os chamados "mundos lexicais" para um determinado grupo social, uma espécie de estrutura do texto produzido por um grupo de sujeitos acerca de um objeto social que demonstra "semântica partilhada pelo grupo" acerca deste mesmo objeto 9 . Os sujeitos podem tomar posições variadas em uma semântica de referência para os vários relatos e sentidos atribuídos pelos indivíduos ao objeto em relação a uma mesma referência semiológica.

Assim, no primeiro nível de análise, o ALCESTE opera na identificação do campo comum (campo consensual), portanto, onde existe alta recorrência e valores de $\mathrm{X}^{2}$ significativos, existe um núcleo de sentido potencialmente válido, que encontram-se demonstrados no dendograma (Figura 1).

No segundo nível, identifica-se o posicionamento dos sujeitos ou grupos face aos campos comuns, que estão ilustrados no plano fatorial (Figura 2).

\section{Resultados e discussão}

O discurso dos sujeitos se apresenta dividido em dois blocos. O primeiro é constituído por quatro classes (1, 3, 4 e 5); com 22\%, 13\%, 21\% e 7\%, respectivamente; as classes 1 e 3 estão fortemente associadas entre si $(R=0,62)$, enquanto as classes 4 e 5 também se associam em uma correlação bastante forte e próxima ( $\mathrm{R}=0,70)$; os dois subconjuntos, por sua vez, correlacionam-se fracamente $(\mathrm{R}=0,32)$ para formar o primeiro bloco. Este bloco foi denominado: As fragilidades dos conselhos de saúde. A classe 2 forma isoladamente o outro bloco, também significativo, dado que congrega $32 \%$ do corpus das falas dos sujeitos, que foi nomeada: A precária organização da Atenção à Saúde.

\section{Classe 1 -}

As limitações no poder de decisão

Esta classe apresenta um discurso típico dos sujeitos do segmento dos prestadores, das Re-

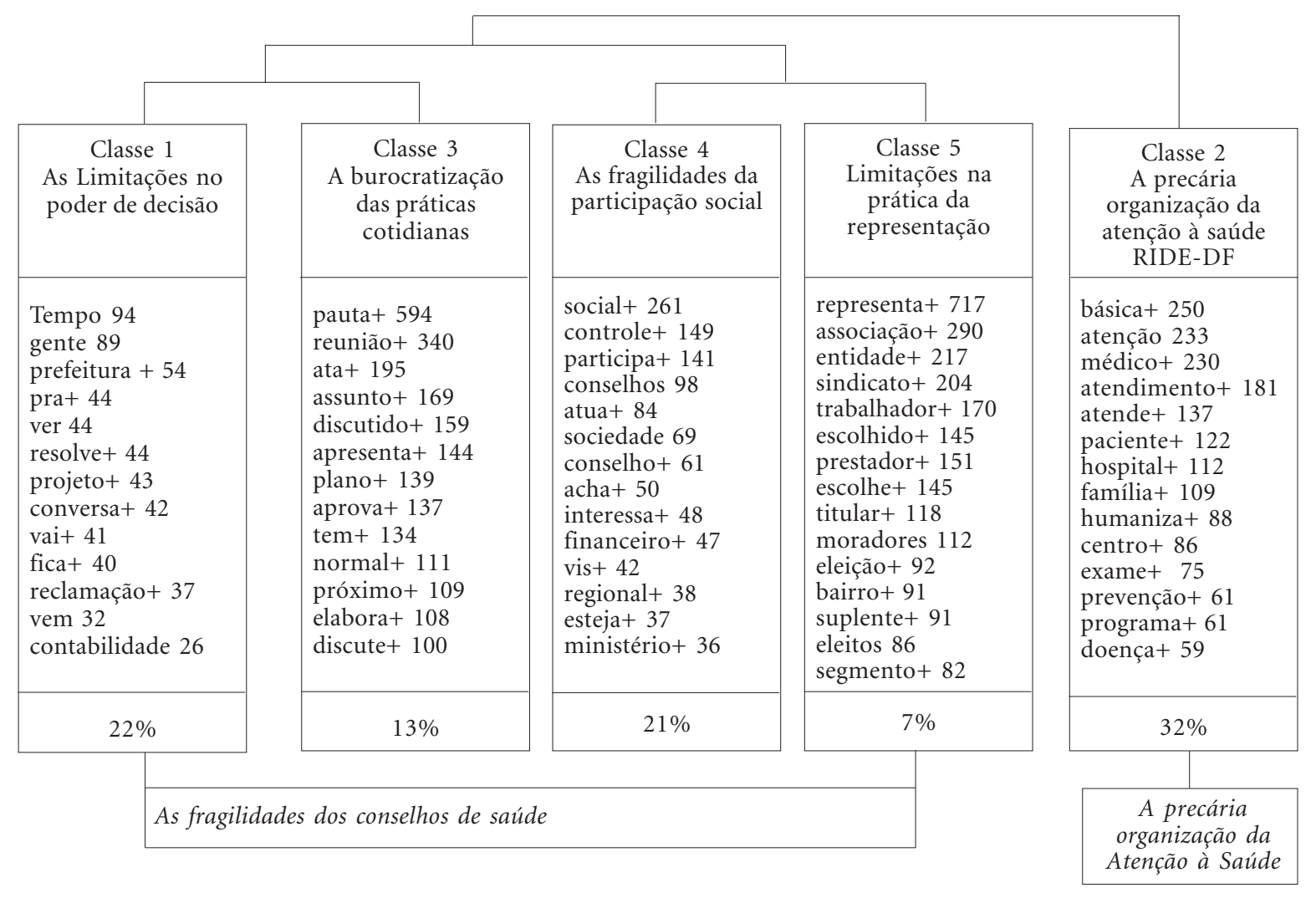

Figura 1. As representações acerca do controle social na RIDE-DF. 


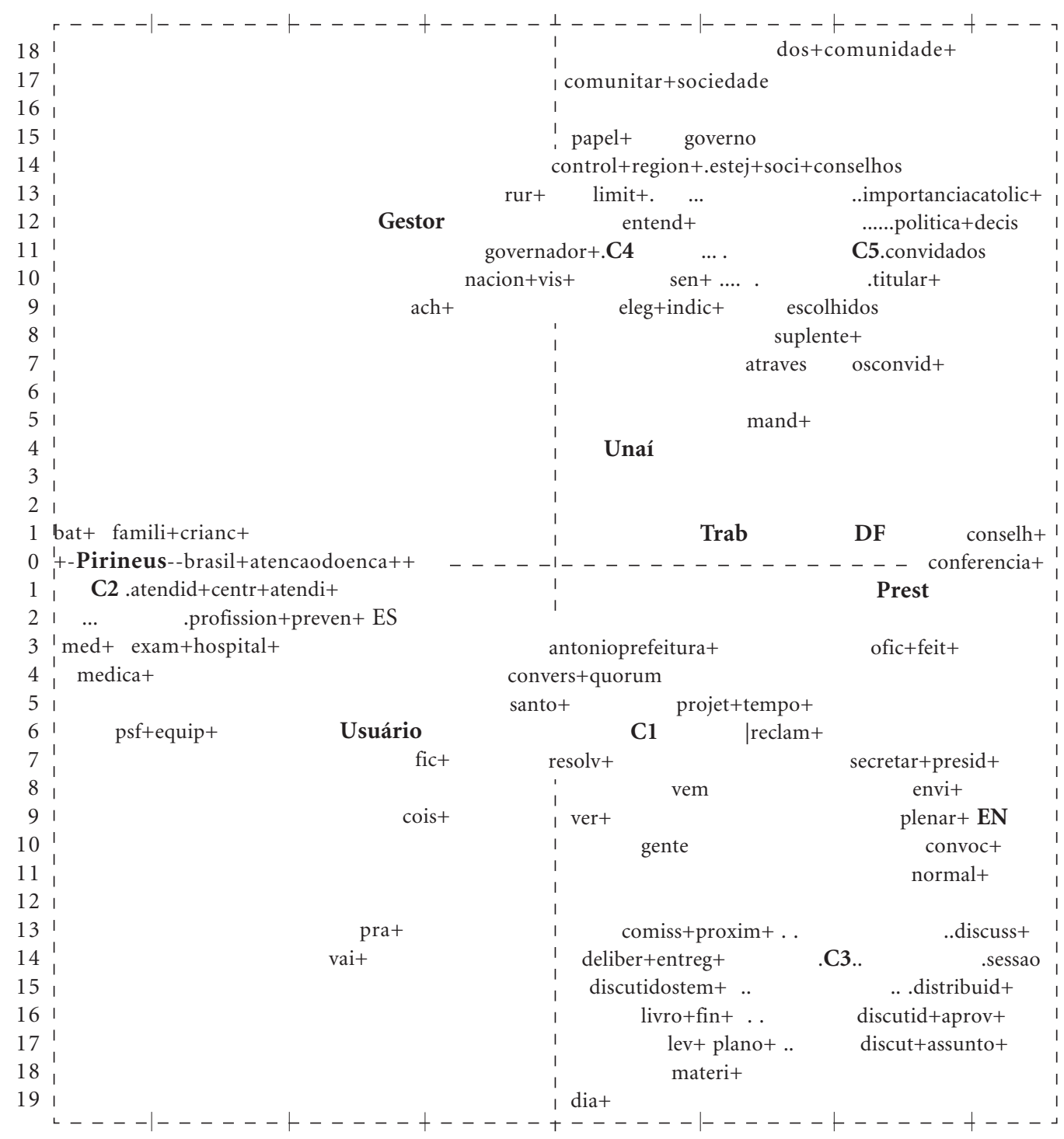

Figura 2. Plano Fatorial com a projeção das variáveis e das palavras na análise fatorial de correspondência sobre controle social em saúde.

giões do Entorno Sul e Pirineus, que trata dos conteúdos das representações relacionadas às principais dificuldades percebidas no cotidiano para a execução dos trabalhos no CS.

Os conselheiros evidenciam que uma das principais funções do conselho é a fiscalização dos serviços de saúde, sobretudo as visitas para analisar as falhas existentes. Contudo, essa atividade não é realizada de modo frequente ou sistemático. A falta de tempo dos conselheiros aparece como principal fator para a não realização das visitas.
Outra atribuição importante assumida pelos conselheiros é a fiscalização das contas públicas, entretanto é sentida como atividade muito difícil e que requer competência técnica específica. Os conselheiros demonstram que há pouco tempo para análise mais detalhada dos gastos, pois passa rapidamente pelo conselho; os sujeitos afirmam ainda que a análise e a avaliação dos orçamentos são realizadas sem muita discussão.

Corroboram para a ocorrência dessa situação a carência de conhecimentos dos conselhei- 
ros do quanto, quando e onde devem ser aplicados os recursos financeiros. Isso ocorre sobretudo porque o "projeto já vem pronto para ser aprovado pela secretaria”, devido à ausência de prática de planejamento participativo.

As relações de poder que se estabelecem entre os representantes do poder executivo e os de usuários, que são em grande parte legitimadas pelo saber técnico se constitui grave problema no interior dos CS. Nessa perspectiva há que se reduzir o poder de quem detém o conhecimento que ainda está localizado na esfera da secretaria municipal. E o poder não pode prescindir da palavra e do diálogo para interferir no espaço público e organizar politicamente os interesses coletivos ${ }^{1}$.

Em suma, nesta classe os conselheiros expressaram o processo de formação das representações sociais com ênfase no controle, por meio de práticas fiscalizatórias, legitimados pelo saber técnico, que coloca o usuário com o seu saber de senso comum, em segundo plano, constrangendo a liberdade de expressão desses sujeitos encarregados de vocalizar as necessidades da população.

A carência das capacitações dificulta que os conselheiros desenvolvam todas as suas funções: a fiscalização do funcionamento do sistema de saúde e dos recursos financeiros; a deliberativa que diz respeito à definição das políticas e prioridades de saúde, a avaliação e deliberação do plano de saúde; e por fim, a função consultiva, que trata da avaliação dos convênios ou outras questões de saúde ${ }^{1,10}$.

\section{Classe 2 -}

A precária organização da atenção à saúde

Esta classe apresenta um discurso típico dos representantes dos segmentos dos usuários e da Região dos Pirineus, que demonstra a percepção da organização da atenção à saúde pelos conselheiros de saúde dos municípios da RIDE-DF, especialmente da Atenção Básica ( $\mathrm{AB})$. Vale lembrar que esta classe está isolada, ou seja, distante das demais, possivelmente porque se trata de conteúdo da representação de controle social que demonstra que a atenção à saúde não tem sido objeto de discussão mais aprofundada nos espaços dos CS.

Verificou-se que na RIDE-DF houve maior investimento dos municípios na expansão da $\mathrm{AB}$ nos últimos tempos, que foi estimulada pelo Ministério da Saúde, inclusive fornecendo aos municípios incentivos financeiros.

Os depoimentos dos conselheiros evidenciaram que a ESF tem se dedicado a realizar atendi- mentos principalmente da demanda espontânea de pessoas com agravos já instalados, porque os municípios, principalmente os de pequeno porte, não possuem serviços de saúde de média e alta complexidade. Além dessa ação, eles evidenciaram que existe oferta organizada de algumas ações ao grupo de materno-infantil, que incluem planejamento familiar e pré-natal. São desenvolvidas também algumas ações de vigilância epidemiológica (Tuberculose, Diabetes, Hipertensão, Hanseníase, dentre outras). Contudo, demonstraram que são incipientes as ações de Vigilância ambiental (falta de tratamento de lixo) e Vigilância Sanitária (controle da qualidade da água).

Verificou-se que existe grande dificuldade para a continuidade do tratamento das doenças, pois os usuários não conseguem realizar os exames básicos nos municípios, e se deparam com grande dificuldade para conseguir atendimento em serviços de média e alta complexidade. Ademais, evidencia-se que o aumento do número de atendimento nas unidades básicas tem ampliado a necessidade de serviços especializados. Como consequência, tem crescido a demanda por investimento na contratação de especialistas, na aquisição de equipamentos mais sofisticados para diagnósticos e tratamento, mas nem todos os municípios conseguem suprir essas demandas, o que tem gerado sentimento de frustração na população.

Nesse particular, cabe ressaltar que as representações dos conselheiros demonstram que os municípios têm ampliado a oferta de serviços básicos, portanto reivindicam com veemência ampliação dos serviços especializados, inclusive implantação de maior número de hospitais. Os conselheiros salientaram ainda a necessidade de expandir os serviços de urgência/emergência, que tem ganhado maior proeminência, face à visibilidade que adquire na mídia em função de pacientes graves sem acesso aos serviços de saúde ${ }^{11}$.

Por fim, há que se salientar que se verifica nas representações dos conselheiros o risco do aprofundamento do modelo hegemônico de atenção à saúde. Portanto, constitui-se desafio trazer para o espaço dos CS a discussão do modelo de atenção que permita desenvolver práticas que acolham, vinculem e resolvam, isto é, promovam e protejam efetivamente a saúde e a vida, no plano individual e coletivo ${ }^{11,12}$.

\section{Classe 3 -}

\section{A burocratização das práticas cotidianas}

Esta classe apresenta um discurso típico dos sujeitos representantes dos trabalhadores da saú- 
de das Regiões do Entorno Norte e do Distrito Federal, que trata das formas de organização do funcionamento cotidiano dos conselhos municipais de saúde.

Os conselheiros evidenciaram que os conselhos estão formalmente organizados para operacionalização das atividades cotidianas, pois têm buscado garantir os encontros entre os representantes: frequência e regularidade dos participantes às reuniões, a organização dos temas para discussão e encaminhamentos, bem como os seus registros.

As tomadas de decisões acerca dos temas a serem debatidos no espaço do conselho são definidas majoritariamente pela secretaria de saúde. Ademais, o grande volume das atividades a serem resolvidos impõe um ritmo intenso de trabalho que reduz a possibilidade de colocarem em discussão as questões relacionadas à formulação de políticas e ações para os municípios.

A forma como são conduzidas as questões relativas à gestão municipal em saúde, apreendida nos depoimentos, sugere que os conselheiros apenas tomam conhecimento, seja das ações e estratégias a serem implementadas, seja do objeto de intervenção, o que corrobora para a construção das representações sociais dos CS como instância burocratizada.

Trata-se de tradição de intervenção coerente com nossa história política, marcada pela centralização de poder, que tem como uma de suas principais facetas a recorrência da verticalidade programática, e de concepções que pretendem fazer do planejamento um conjunto de decisões técnicas, como se técnica pudesse ser dissociada de seus efeitos políticos de produção da realidade ${ }^{3,13-15}$.

São essas informações que definem todas as ações e a forma de organização do serviço, sem nenhuma consulta às demandas dos usuários que buscam os serviços de saúde. A população não é considerada como sujeito político, mas como objeto, como número que se apresenta em cálculos e em tentativa de previsão e ordenação da assistência ${ }^{15}$.

Essa forma de organização das práticas no conselho, extremamente centralizada e burocratizada, impede que os sujeitos dialoguem com sua experiência e com os desejos nele implicados, revelando-se pouco porosos à produção de novas possibilidades de construção de projetos coletivos $^{15}$.
Classe 4 -

As fragilidades da participação social

Esta classe apresenta um discurso típico dos sujeitos representantes dos trabalhadores da Região de Unaí e do Distrito Federal, que expressaram conteúdo de representações que têm como eixo central a participação social na formulação, no monitoramento e na avaliação das políticas municipais. Na visão dos conselheiros, essa participação requer o desenvolvimento de um conjunto de práticas que subsidiem satisfatoriamente a organização das políticas e dos serviços de saúde nos municípios com base nas demandas e necessidades da população local, mas que ainda encontra-se embrionária nos municípios.

O controle social entendido como o controle da sociedade civil sobre as ações do Estado se encontra ancorado na utopia igualitária idealizada dentro do processo da Reforma Sanitária, com o intuito de garantir a saúde como um direito do cidadão e a construção do fortalecimento do poder local por meio de uma gestão social democrática $^{3,12}$. Nessa perspectiva, o fortalecimento do exercício do controle social sobre o Estado permitiria a expansão da esfera pública, por meio da criação de canais de participação social ${ }^{16}$.

Todavia, reiterou-se nesta classe que é forte a conformação da representação social do controle social como a participação social no controle e/ou fiscalização das ações e políticas da saúde, sob o argumento de que o CS impõe ao gestor de saúde a necessidade de maior transparência e rigor em relação às ações desenvolvidas no município.

Essa representação encontra ancoragem no contexto de um fenômeno político denominado accountability, que se trata de um espaço político onde um ou diversos atores sociais podem cobrar publicamente de outros (instituições, organizações empresariais, autoridades ou lideranças políticas) comportamentos geradores de transparência e de adequação de seus atos às expectativas de interesse individual, público e/ou coletivo, de acordo com as normas e funções previstas $^{1}$. Nos CS da RIDE-DF a prática do accountability está mais voltada para o controle das contas públicas, que é feito de forma burocrática, sem compreensão aprofundada desse processo pelos diversos atores envolvidos.

Embora a participação social em saúde seja reconhecida pelos conselheiros como um direito 
do cidadão, constituindo-se importante canal para o exercício da cidadania e da afirmação de novos direitos, ou seja, que não se limita à garantia de direitos previamente definidos pelo Estado, mas na conquista de outros emanados das práticas concretas ${ }^{15}$, deparam-se com a falta de permeabilidade para interferirem mais incisivamente no espaço da gestão em saúde.

Contribui para a fragilidade do controle social em saúde a falta de articulação do CS com a população local, que tem baixa cultura de participação na formulação de propostas para resolução dos problemas de saúde. A forma como o CS tem se portado junto à população também tem contribuído para o distanciamento entre ambos, ou seja, o desconhecimento dos conselhos: dos seus objetivos, das suas funções e mesmo das suas utilidades. Nessa direção, os conselheiros apontam a importância da articulação entre os CS, bem como conselhos de outras áreas sociais para avançar no processo de gestão participativa em saúde.

\section{Classe 5 -}

\section{Limitações na prática da representação}

Esta classe apresenta um discurso que foi típico dos sujeitos do Distrito Federal, na qual são tratados assuntos como a composição, a representação e a representatividade possibilitados pelo CS. São conteúdos de representações do controle social que tratam dos limites da democracia representativa nos espaços dos CS.

É fundamental que a escolha das organizações sociais para comporem o conselho seja realizada em fórum próprio da sociedade civil a fim garantir a legitimidade das instituições que participam perante um espaço público ${ }^{17}$. Nesse sentido, verificou-se que são poucos os municípios da RIDE-DF que adotam postura mais democrática, ou seja, que fazem a eleição dos representantes do conselho em Conferência municipal. A indicação e a escolha dos conselheiros em assembleias abertas atenua a interferência de prefeitos e secretários de saúde e, ainda, pode induzir as associações a democratizarem-se mais, já que muitas vezes reproduzem em seu interior os mesmos resquícios autoritários e excludentes que atravessam a sociedade como um todo ${ }^{18}$.

O tempo de permanência dos conselheiros da RIDE-DF também merece destaque, pois parece prevalecer uma tendência ao "profissionalismo", já que com frequência as mesmas pessoas são reconduzidas ao cargo por seu carisma e conhecimento dos trâmites do conselho. Não obs- tante, é razoável deduzir que nos municípios menores, os líderes comunitários e as pessoas com mais experiência sejam os escolhidos para atuar nos CS, reforçando a formação de uma "carreira profissional" de conselheiro de saúde.

A ausência de organização política dos setores excluídos obviamente reforça essa dificuldade, mais notadamente nos municípios de pequeno porte da RIDE-DF. O risco é de que setores da classe média (Rotari, Lions, maçonaria) e quadros técnicos de suas entidades passem a ser porta-vozes daqueles segmentos, o que desfigura a representatividade dos CS.

Um conselho só é representativo quando agrega uma diversidade de segmentos da sociedade civil e, portanto, uma série de distintas entidades a serem representadas no $\mathrm{CS}^{19}$. A fragilidade dos movimentos, que nos últimos anos sofreram um esvaziamento importante do seu papel político de representação e articulação de demandas ${ }^{14}$, repercute diretamente na conformação dos conselhos.

Nessa lógica, tem sido discutida a importância da formação de um capital social, um dos elementos que conformam uma comunidade cívica, em que o associativismo se organiza de forma horizontal, não centralizada, a fim de que seus membros sejam sujeitos de novas relações sociais, baseadas nos valores de solidariedade, dos direitos e do interesse público ${ }^{17}$.

Há que se destacar que os conselhos tornamse virtuosos na medida em que são percebidos como espaços de decisão construídos pela capacidade efetiva de atuação da representação popular da coletividade ${ }^{20}$. Assim, faz-se necessário buscar outros espaços de escuta das demandas da população a fim de identificar, inclusive, outros modos de participação mais ativos de atores diversos em diferentes arenas de decisão e em permanente negociação.

\section{Análise Fatorial de correspondência}

A análise fatorial de correspondência permite observar as diferenças de posicionamento grupais, possibilitando uma complementação das informações apontadas na classificação hierárquica descendente. Estão distribuídas nos diferentes quadrantes, de forma não aleatória, as classes com a projeção de algumas palavras, bem como as variáveis suplementares.

No quadrante superior do plano fatorial se verifica os discursos dos conselheiros do DF e da Região de Unaí que estão associadas às classes $4 \mathrm{e}$ 5 , e que tratam das representações sociais relacionadas às fragilidades da participação institucio- 
nalizada. E no quadrante inferior encontram-se os discursos dos conselheiros das regiões do Entorno Norte, Entorno Sul e Pirineus que estão associadas às classes 1,2 e 3 , demonstrando uma maior organização do CS, embora burocratizado, com participação mais ativa dos conselheiros, especialmente do usuário, no exercício do controle social em saúde.

Da distribuição dos elementos no plano fatorial é possível apreender duas dimensões acerca da representação do controle social. A primeira está relacionada ao segmento representado no conselho, observa-se que o gestor e o trabalhador vocalizam a organização formal do conselho, contribuindo para a conformação das representações sociais do controle social com ênfase na burocratização.

Deve ser reiterado que a atenção à saúde não é objeto de análise principal dos CS porque existem diversos outros pleitos para serem discutidos, impressos pelo modo burocratizado de funcionamento dos CS. Como já foi dito, contribui especialmente para o distanciamento do CS do seu foco principal a postura centralizadora dos gestores. O segmento dos usuários é que tenta trazer para o CS a discussão das necessidades da população. Há ênfase em reivindicar por mais serviços especializados (consultas, exames, cirurgias), com o argumento de que a população não pode pagar planos de saúde privados. Além disso, expressaram a necessidade de construção de mais hospitais nos municípios devido a dificuldade de resolver problemas de saúde que requerem tecnologias de alta densidade que, comumente, são encaminhados aos municípios maiores ou à Brasília (DF).

A segunda dimensão é relativa à capacidade organizativa da sociedade civil de acordo com as características demográficas, econômicas e políticas das regiões. A região do Entorno Sul e Entorno Norte são compostas por municípios que possuem precária infraestrutura e o nível de pobreza da população é significativo. Contudo, é uma área que tem se desenvolvido no processo de urbanização e com maior riqueza de grupos sociais organizados e politizados ${ }^{21}$. Possui conselheiros com um pouco mais de "voz política", ou seja, maior capacidade para expressarem sobre os direitos de participação social no espaço do conselho. Nessa direção, percebem criticamente a precariedade das condições da organização e funcionamento dos conselhos para o exercício do controle social.

\section{Considerações Finais}

A representação dos conselheiros de saúde da RIDE-DF acerca do controle social, ainda em processo de formação, demonstra a burocratização dos CS, sobretudo porque as gestões municipais ainda estão ancoradas em modelo bastante centralizador, que parecem ser refratárias ao controle social e agem buscando reduzir o seu papel deliberativo e fiscalizador a rituais de legitimação de políticas por eles elaboradas.

Ressalta-se que a forma de organização e funcionamento dos CS, hierarquizada e burocratizada, tem distanciado os conselheiros das discussões sobre as necessidades de saúde, que deveriam ser o foco principal, para a construção de Projeto de Saúde voltados para os interesses das coletividades.

De fato, a institucionalização da participação social nos conselhos é permeada por interesses conflitantes e contraditórios, no qual a defesa da questão pública coloca-se em destaque; neste estudo, foram os usuários que, apesar dos constrangimentos a que estão submetidos no âmbito CS, tentaram vocalizar a discussão da atenção à saúde, ainda que na perspectiva do modelo hegemônico

Os conselhos como espaços de participação e controle social nas ações do Estado podem ser revinventados, buscando ouvir a população, os seus desejos, seus modos de viver e agir, permitindo a livre expressão das demandas e necessidades de saúde da população. 


\section{Colaboradores}

HE Shimizu trabalhou na concepção e no delineamento do estudo, na análise e na interpretação dos dados, na redação e na revisão final do artigo. MF Pereira participou da concepção e do delineamento do estudo, e da coleta de dados. XPCD Bermudez participou da discussão dos dados. AJCC Almeida da discussão dos dados e revisão do artigo.

\section{Agradecimentos}

Aos conselheiros de saúde dos municípios da RIDE-DF que forneceram as informações sobre a dinâmica de funcionamento dos conselhos. Ao $\mathrm{CNPq}$ pelo financiamento do estudo. E aos alunos e aos professores do Departamento de Enfermagem da UnB que participaram da coleta de dados.

\section{Referências}

1. Oliveira VC. Comunicação, informação e participação popular nos conselhos de saúde. Saúde $e$ sociedade 2004; 13(2):56-69.

2. Acioli S. Participação social na saúde: revisitando sentidos, reafirmando propostas. In: Pinheiro R, Mattos RA, organizadores. Construção social da demanda: direito à saúde, trabalho em equipe, participação e espaços públicos. Rio de Janeiro: IMS/ UERJ, Abrasco; 2005. p. 293-303.

3. Acioli GG. Das dimensões pedagógicas para a construção da cidadania no exercício do controle social. Interface (Botucatu) 2007; 11(23):409-425.

4. Santos Junior AO, Azevedo S, Ribeiro LCQ. Democracia e gestão local: a experiência dos conselhos municipais no Brasil. In: Santos Júnior AO, Ribeiro LCQ, Azevedo S, organizadores. Governança democrática e poder local: a experiência dos conselhos municipais no Brasil. Rio de Janeiro: Revan; 2004. p. 11-56.

5. Santos BS, Avritzer L. Para ampliar o cânone democrático. In: Santos BS, organizadores. Democratizar a democracia: os caminhos da democracia participativa. 3a Edição. Rio de Janeiro: Civilização Brasileira; 2005.

6. Jodelet D. O movimento de retorno ao sujeito e a abordagem das representações sociais. Sociedade $e$ Estado 2009; 24(3):679-712.

7. Abric JC. A abordagem estrutural das representações sociais. In: Moreira ASP, Oliveira DC, organizadores. Estudos interdisciplinares de representação social. Goiânia: AB editora; 1998.

8. Reinert M. Une méthodologie d'analyse des données textuallles et une aplication. Bulletin de Sociologie 1990; 26(9):24-54.

9. Guimarães SP, Campos PHF. Norma social violenta: um estudo de representação social da violência em adolescentes. Psicol reflex crít 2007; 20(2):188-196.

10. Brasil. Ministério da Saúde (MS). Conselho Nacional de Saúde. Resolução no 333, de 4 de novembro de 2003. Aprova as seguintes diretrizes para criação, reformulação, estruturação e funcionamento dos Conselhos de Saúde. Diário Oficial da União 2003; 4 dez.

11. Paim JS. Desafios para a saúde coletiva no século XXI. Salvador: EDUFBA; 2006.

12. Merhy EE. A rede básica como construção da saúde pública e seus dilemas In: Merhy EE, Onocko R, organizadores. Agir em saúde: um desafio para o público. São Paulo: Hucitec; 1997.

13. Paim JS, Teixeira CF. Configuração institucional e gestão do Sistema Único de Saúde: problemas e desafios. Cien Saude Colet 2007; 12(Supl.):1819-1829.

14. Gerschman S. Conselhos municipais de saúde: atuação e representação das comunidades populares. Cad Saude Publica 2004; 20(6):1670-1681.

15. Machado FR, Pinheiro R, Guizardi FL. Direito à saúde e integralidade no SUS: o exercício da cidadania e o papel do Ministério Público. In: Pinheiro R, Mattos R, organizadores. Construção social da demanda, direito à saúde, trabalho em equipe, participação e espaços públicos. Rio de Janeiro: CEPESC, UERJ, ABRASCO; 2005. 
16. Gohn MG. Os conselhos municipais e a gestão urbana. In: Santos Junior AO, Ribeiro LCQ, Azevedo $\mathrm{S}$, organizadores. Governança democrática e poder local: a experiência dos conselhos municipais no Brasil. Rio de Janeiro: Revan; 2004. p. 57-93.

17. Santos MRM. A representação social no contexto da participação institucionalizada: o caso dos conselhos municipais do Rio de Janeiro. In: Santos Junior AO, Ribeiro LCQ, Azevedo S, organizadores. Governança democrática e poder local: a experiência dos conselhos municipais no Brasil. Rio de Janeiro: Revan; 2004.

18. Labra ME, Giovanella L. Estudio de caso: Brasil, construcción del Sistema Único de Salud y participación de la sociedad civil. Rio de Janeiro: Fiocruz, Red de Investigación en Sistemas y Servicios de Salud del Cono Sur; 2007.

19. Machado EHH, Batista JV, Kornin T. Governança urbana: um estudo sobre os conselhos municipais da Região Metropolitana de Curitiba. In: Santos Junior AO, Ribeiro LCQ, Azevedo S, organizadores. Governança democrática e poder local: a experiência dos conselhos municipais no Brasil. Rio de Janeiro: Revan; 2004.

20. Caccia BS. Os conselhos como instrumentos da sociedade. In: Teixeira ACC, Albuquerque MC, organizadores. Conselhos gestores de políticas públicas. São Paulo: Instituto de Estudos, Formação e Assesoria em Políticas Sociais; 2000. (Revista Pólis, N. 37).

21. Menicucci T. A implementação da reforma sanitária: a formação de uma política. In: Hochman G, Arretche M, Marques E, organizadores. Políticas públicas no Brasil. Rio de Janeiro: Fiocruz; 2007.

Artigo apresentado em 07/08/2012

Aprovado em 19/10/2012

Versão final apresentada em 08/11/2012 